\title{
Transience Analysis of Bursty Traffic with Erbium Doped Fiber Amplifiers
}

\author{
C. Reis*, B. Neto*, R. Dionisio*, G. Incerti ${ }^{+}$, G. Tosi-Beleffi ${ }^{+}$, D. Forin ${ }^{+}$, A. M. Rocha* \\ A. L. J. Teixeira*, P. S. André* \\ * Instituto de Telecomunicações, Campus universitário de Santiago3810-193 Aveiro, Portugal \\ Tel: (+351) 234377900, Fax: (+351) 234377901 \\ e-mail: \{creis, bneto, rdionisio, amrocha, teixeira, pandre\}@av.it.pt, \\ ${ }^{+}$Instituto Istituto Superiore Delle Comunicazioni e Delle Tecnologie Dell' Informazione (ISCOM) \\ Viale America, 201-00144 Roma - Italia \\ e-mail: \{gabriele.incerti, giorgio.tosibeleffi, davide.forin\}@comunicazioni.it,
}

\begin{abstract}
In this paper, we investigate experimentally the impact of optical amplifiers, namely Erbium Doped Fiber Amplifiers (EDFA) on Optical Burst Switching (OBS) networks, operating at $2.5 \mathrm{~Gb} / \mathrm{s}$ (typical bit rate for current passive optical networks - PON). Bursts with $2^{12}-1$ and $2^{16}-1$ pseudo random binary sequence (PRBS) were studied, with different idle times (from $2 \mu \mathrm{s}$ to $80 \mu \mathrm{s}$ ). The use of traffic with variable idle time was assessed in order to evaluate the effect on the burst initial amplitude. We also analyzed the performance of the packet based system by measuring the $\mathrm{Q}$ factor at the receiver and concluded that the performance is not affected by the type of traffic (variable or fixed idle times).
\end{abstract}

Keywords: optical burst switching (OBS), transience, optical amplifiers, erbium doped fiber amplifiers (EDFA).

\section{INTRODUCTION}

The gain dynamics of optical amplifiers is a topic of considerable interest in wavelength division multiplexed (WDM) networks, as result of the adding and dropping of channels in reconfigurable networks but also due to the use of bursty traffic, where data bearing signals are turned on and off in different time scales. In this kind of traffic, data packets are assembled into bursts to the WDM channel and transported across the optical core to the destination. An inherent aspect of this transmission technology is the existence of long inter-burst idle intervals (from nanoseconds to seconds) that can enhance the transience due to optical amplifiers [1, 2]. It must be noted that the transient is cumulative as it propagates through multiple amplifiers, and thus its associated time constant becomes shorter which can jeopardize seriously the clock and data recovery at the receiver [3].

The Erbium doped fibre amplifier (EDFA) transients arise due to the long upper state lifetime of Erbium ions and the deep saturation operation of such devices. For packet based networks, if the input packet power is high and its duration is long, the population inversion of Erbium ions experiences a quick depletion causing a fast power transient across the packet [4]. The restoration of the initial conditions depends on the idle time. If the latter is high enough to allow a complete inversion of population of Erbium ions, the initial conditions are restored and the next packet will behave as its precedent. However, if the next packet arrives before complete inversion, it will experience less initial gain and consequently less power decay. The main idea of this experiment is to assess the amount of gain excursion with variable idle time in order to evaluate how consecutive packets are affected by variable idle time. The system performance is also appraised by the Q factor analysis with the packet density. The paper is organized as follows: in section 2, we described the experimental work that was carried out, in section 3 the main results are presented and discussed and finally, in section 4, the conclusions are drawn.

\section{EXPERIMENTS}

The implemented experimental setup consists of an external cavity laser peaking at $1549.32 \mathrm{~nm}$ and $10 \mathrm{dBm}$ of optical power followed by a polarization controller and a Mach-Zehnder optical modulator at $2.5 \mathrm{~Gb} / \mathrm{s}$. The considered data are programmable packets produced by a BERT (Agilent N4901B). The optical signal is then amplified in an EDFA (IPG-EAD-500-C3-W), attenuated and then injected into a span of $40 \mathrm{~km}$ of SSMF. The fiber input and output powers are $10 \mathrm{dBm}$ and $0.5 \mathrm{dBm}$, respectively. As receivers, a PIN (HP-11982A) and an (Agilent Infinium 86100A) oscilloscope were used. The EDFA was previously characterized in order to determine the threshold driving current of its pump $(0.7 \mathrm{~A})$ and the signal saturation power $(-10 \mathrm{dBm}$ for a pump driving current equal to $1.98 \mathrm{~A}$ ). It must be noted that all the subsequent experiments were carried out with the EDFA in deep saturation (pump driving current equal to $1.98 \mathrm{~A}$ ). The packets were programmed in order to have a constant length (with $2^{12}-1$ and $2^{16}-1$ for each studied situation). The idle times were varied according to the sequences described in Figure1 


\begin{tabular}{|c|c|c|c|c|}
\hline Seq. A & $\begin{array}{l}2^{12}-1 \\
\text { PRBS }\end{array}$ & $\begin{array}{c}10000 \\
" 0 "\end{array}$ & $\begin{array}{l}2^{12}-1 \\
\text { PRBS }\end{array}$ & $\begin{array}{c}50000 \\
" 0 "\end{array}$ \\
\hline Seq. B & $\begin{array}{l}2^{12}-1 \\
\text { PRBS }\end{array}$ & $\begin{array}{c}100000 \\
" 0 "\end{array}$ & $\begin{array}{l}2^{12}-1 \\
\text { PRBS }\end{array}$ & $\begin{array}{c}200000 \\
" 0 "\end{array}$ \\
\hline Seq. $C$ & $\begin{array}{l}2^{16}-1 \\
\text { PRBS }\end{array}$ & $\begin{array}{c}10000 \\
" 0 "\end{array}$ & $\begin{array}{l}2^{16}-1 \\
\text { PRBS }\end{array}$ & $\begin{array}{c}50000 \\
" 0 "\end{array}$ \\
\hline & $\begin{array}{l}2^{16}-1 \\
\text { PRBS }\end{array}$ & $\begin{array}{c}100000 \\
" 0 "\end{array}$ & $\begin{array}{l}2^{16}-1 \\
\text { PRBS }\end{array}$ & $\begin{array}{c}200000 \\
" 0 "\end{array}$ \\
\hline
\end{tabular}

Figure 1. Programmed bit sequences.

\section{RESULTS}

The patterns of the packets with $2^{12}-1$ (Seq. A and Seq. B) and $2^{16}-1$ (Seq. C and Seq. D) were visualized in the oscilloscope as displayed in Fig. 2 and Fig. 3, being noticeable that the packets exhibit different gain excursion due to the variable idle time. The initial power difference of consecutive packets was then computed. For the lower length packets $\left(2^{12}-1\right.$ PRBS $)$ that difference decreases with the idle time $(21.81 \mu$ s for Seq. A and $3.76 \mu$ s for Seq. B). This result was interpreted by considering that lower idle time situation is not sufficient to obtain a good inversion of population of Erbium ions. The same tendency was also observed for the longer length packets (Seq. C and Seq. D).
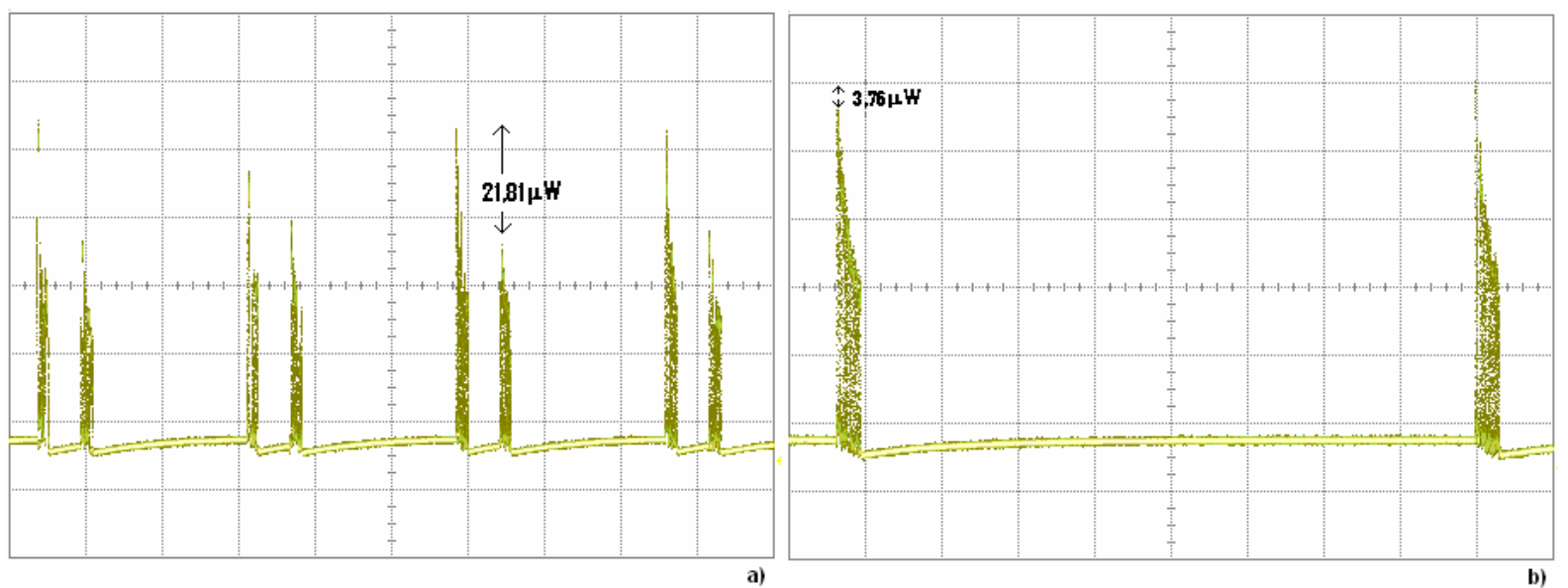

Figure 2. Visualized sequences at the receiver: (a) Seq. A, (b) Seq. B. Horizontal scale $5.000 \mu$ s/div and vertical scale is arbitrary.

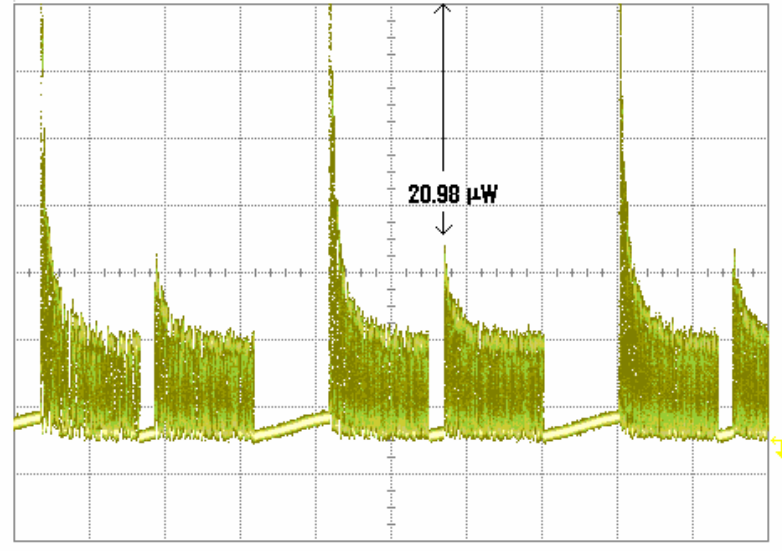

a)

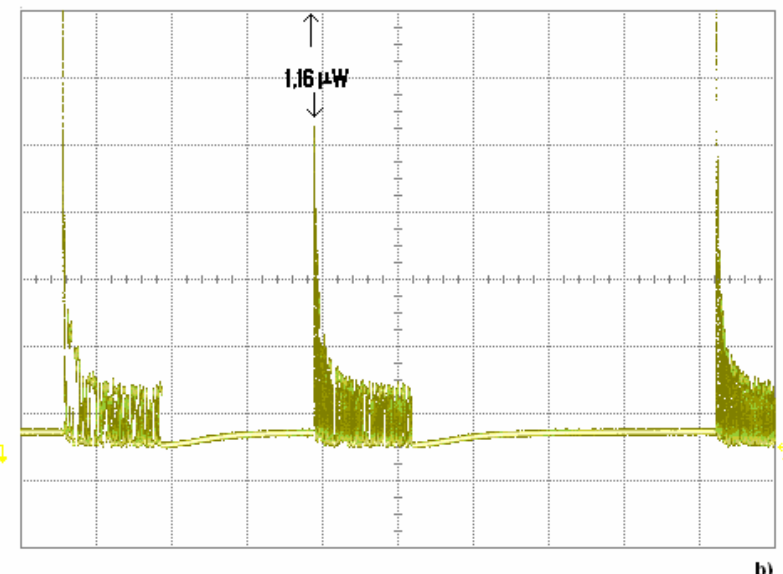

b)

Figure 3. Visualized sequences at the receiver: (a) Seq. C, (b) Seq. D. Horizontal scale $20.00 \mu$ s/div and vertical scale is arbitrary.

To assess the performance of a network in the presence of packet traffic, we have analyzed the $Q$ factor at the receiver and compare its value with the $Q$ factor in the back to back situation. The $Q$ factor difference between the back-to-back and the receiver $(\Delta Q)$ was computed as a function of the packet occupancy density, as depicted in Figure 4. The used sequences include packets with $2^{16}-1$ intercalated with variable idle times in the $2-80 \mu \mathrm{s}$ range (points 1, 3, 5 and 7 in Figure 4) and packet with $2^{16}-1$ intercalated with fixed idle times in the $2-80 \mu \mathrm{s}$ 
range. By looking at the plot, we notice that the best situations, the ones in which the $\Delta Q$ has a lower value (points 6,7 and 8), is obtained with a high packet density. This result is due to the increase in the average power at the receiver. We also notice that the $\Delta Q$ is quite stable in the $0.3-0.7$ occupancy density range and that the gain excursion due to variable idle time does not seem to be more penalizing than the situation with fixed idle time.

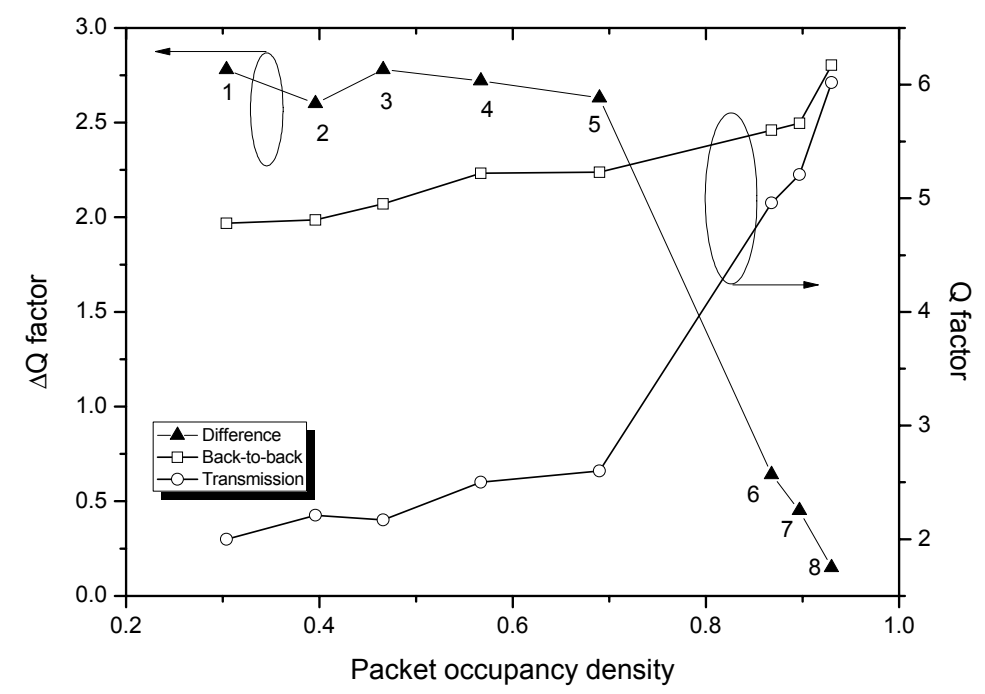

Figure 4. Qfactor as a function of the occupancy density. The lines are visual guides.

\section{CONCLUSIONS}

We have studied the effect of EDFA transience with packet based traffic with variable idle time. Thus, we assess experimentally the amount of gain excursion with variable idle time and concluded that it decreases with higher idle times. This result matches with the theoretical expectation that higher idle time restore the inversion of population of the Erbium ions in the EDF.

We have also studied the performance of the system with different types of traffic (variable and fixed idle times) and concluded that a maximum impact factor $(\Delta Q)$ of 2.8 is obtained.

\section{ACKNOWLEDGEMENTS}

This work was supported by the POSC program (FEFOF projects), by the European Union EURO-FOS and SARDANA projects B. Neto and A. M. Rocha gratefully acknowledges their Ph. D. grants financed by FCT (SFRH/BD/28904/2006 and SFRH/BD/41773/2007 respectively).

\section{REFERENCES}

[1] C. Reis, A. M. Rocha, B. Neto, N. Wada, P. André: Raman Amplification in high 10 Gbit/s and 40 Gbit/s Packet Optical Networks, in Proc. ICTON 2008-Mediterranean Winter, Marrakech, Marrocco, Dec. 2008.

[2] P. André, B. Neto, A. Teixeira, N. Wada: Raman amplification impact in packet base networks, Microwave and Optical Technology Letters 50, pp. 3083-3085 (2008).

[3] Y. Awaji, H. Furukawa, N. Wada, P. Chan, R. Man: Mitigation of the transient response of erbium doped fiber amplifiers for burst traffic high speed optical packets, in Proc. CLEO, JTuA133, 2007.

[4] A. Bononi, L.A. Rusch: Doped-fiber amplifier dynamics: a system perspective, J. Lightwave Technol. 16 pp. 945-956 (1998). 